\title{
Designing a Low-Cost Mobile Tracking System for Communication with a Medium Earth Orbit Satellite
}

\author{
Peter Dong, Harrison Carcione, Joseph Hutter, Gina \\ Jiang, Charles Kuch, Soomin Park, Advai Podduturi, \\ Peijing Xu \\ Illinois Mathematics and Science Academy \\ Aurora, IL, USA 60506 \\ \{pdong, hcarcione, jhutter, gjiang, ckuch, spark3, \\ apodduturi,pxu\}@imsa.edu
}

\author{
David Beering \\ Intelligent Designs, LLC \\ Wheaton, IL, USA 60189 \\ drbeering@sprynet.com
}

\begin{abstract}
An essential part of satellite communication is the orientation of the antenna, which can be difficult to ascertain on mobile platforms such as ships. While equipment to measure orientation accurately at sea exists, current solutions are expensive. This paper describes work toward an antenna orientation system using low-cost Global Position System (GPS) receivers. We investigated two methods: one using the spatial difference between multiple GPS units at the vertices of a polygon, and the other using the differences over time measured using a single GPS unit.

We tested the antenna orientation system with the Omnispace F2 satellite at the US Electrodynamics, Inc. (USEI) teleport in Brewster, WA. Although non-correlated systematic errors in the GPS receivers made the multiple-GPS system impractical, the time-differential method was able to maintain a satellite lock for the majority of a simple test course. The reliability of this solution may be further improved using a gain-based correction algorithm.
\end{abstract}

Keywords-GPS; heading determination; orientation model; satellite communication

\section{INTRODUCTION}

Satellite communication is essential for mobile platforms, especially naval vessels. Smaller vessels would benefit from a satellite communications system that is small, easy to install, and inexpensive. For low and medium Earth orbit satellites, the antenna mount has to turn to follow the satellite's trajectory, which requires knowledge not only of the satellite's ephemeris data, but also the boat's position and heading and an exact time reference. However, while Global Positioning System (GPS) units make it easy to find the position of the vessel and the time, finding its bearing is difficult. While all vessels have instruments that measure bearing, connecting to those instruments would be complicated and vessel-specific. A satellite tracking system that can determine orientation independently of the vessel would be easier to install, more flexible, and more robust.

Unfortunately, GPS receivers have limited precision, thus making determination of orientation difficult. There are differential solutions which improve GPS precision to the level needed to determine heading using real-time kinematics (RTK), but these require high-quality antennas and sophisticated circuitry to measure carrier wave phase difference. Existing solutions are also expensive [1]. Precise point positioning (PPP) methods are only useful for postprocessed data, making them useless for this application [2]. We tested two methods for determining bearing using low-cost equipment: one that uses multiple GPS receivers to improve precision, and one that uses multiple measurements over time by a single GPS receiver to determine heading.

We tested our system using Omnispace F2 (formerly ICO F2), a medium Earth orbit satellite with an altitude of 10,500 $\mathrm{km}$ and an inclination of 45 degrees to the equator. We used a helical antenna attached to a rotating mount with motor controllers that can point the antenna toward any location in the sky with two axes of motion: azimuth and elevation. The antenna has a $3-\mathrm{dB}$ beam width of about 60 degrees. Our goal was to maintain a high received signal strength from the satellite as the test platform changed direction. This was measured using $\mathrm{E}_{\mathrm{b}} / \mathrm{N}_{0}$ (energy per bit to noise power spectral density ratio) as reported by the satellite modem.

\section{Design OF THE HeAdING UNIT}

We started by considering three methods for determining orientation, of which the simplest solution used a GPS unit and an inexpensive magnetometer to find bearing. The bearing was corrected using a magnetic declination map. However, we discovered that magnetic fields as small as $0.045 \mathrm{mT}$ interfered with the magnetometer substantially, giving highly unreliable results. Since we encountered much larger magnetic fields in the laboratory and outside during our tests, we concluded that the magnetometer was not a robust solution.

This left us with two approaches: using multiple GPS receivers to determine heading by the difference in position between different receivers, and using the time differential between multiple readings from a single receiver to determine the heading of a moving vessel.

\section{A. Multiple-GPS Method}

A simple method for determining heading is to use the difference between the positions of two GPS receiver. The angle of the vector connecting the two positions can be measured with respect to true north. However, to keep the system easy to install, the GPS units need to be close to each other, at which point their limited precision introduces large errors into the calculation of the heading. This problem can be mitigated by the addition of more GPS units; however, this complicates the calculation of the heading. 


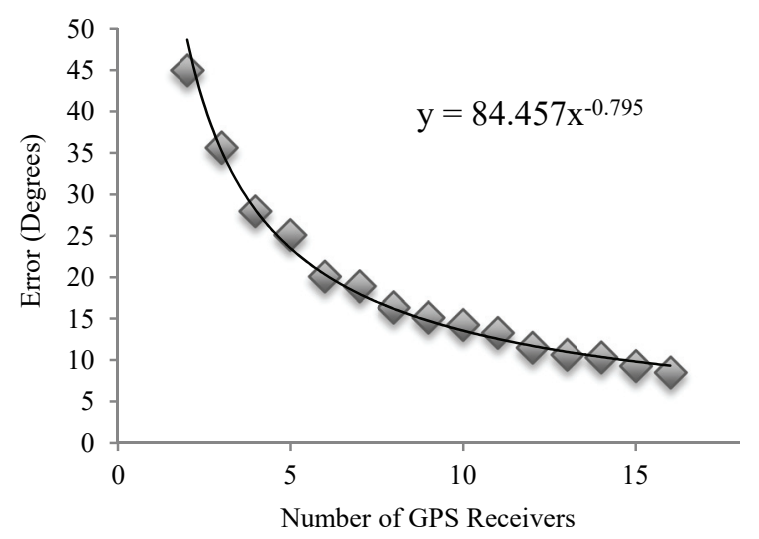

Fig. 1. Error in heading determination of computer-generated GPS positions. We created computer-simulated GPS units at the vertices of a regular $n$-gon. GPS-recorded positions were generated using a uniform distribution within a radius equal to the radius of the polygon. For each point, we rotated and translated the polygon to minimize the squares of the distances to the measured positions. The resulting polygon gave a heading, and the error was the difference between this heading and the true value. Each data point shows the average of 100 trials.

The GPS receivers are positioned in a known shape with an unknown orientation. Our algorithm takes the known shape and size of the receivers and rotates and shifts it to get as close as possible to the recorded positions by minimizing the $\chi^{2}$, which is the square of the difference between the measured positions and the theoretical position of the receives. The direction of the best-fit polygon determines the heading.

To test the effect of the number of GPS receivers on position, we simulated many trials using GPS receivers placed at the vertices of a regular $n$-gon. We simulated measured GPS positions by using a uniform error within a radius equal to the radius of the polygon. The predicted improvement in the error for more GPS units is shown in Fig. 1. After considering the feasibility of constructing and mounting GPS units on a physical structure, we decided to use four GPS units for our tests.

One problem that arose was that four GPS units rarely gave four different readings, and if two gave the same position reading the fitting algorithm did not work. To deal with this, we extrapolated values from a sliding window of 10 measured positions and used a linear extrapolation to estimate the position for the current time.

The test structure consisted of four Adafruit Ultimate GPS Logger Shields (\$44.95 each), each connected to an Arduino Uno microcontroller (\$24.95 each). One unit was secured to each end of each of two wooden boards, which were bolted together at right angles, and foam padding was placed beneath the circuit boards to minimize the effects of vibration (Fig. 2). We found in testing that a GPS unit could detect differences in position of about $2.3 \mathrm{~m}$, so we used 2.5 -m boards.

A USB hub at the center of the boards connected the GPS receivers to a computer. The unit was tested by placing the structure on a car and driving slowly along a predetermined route. The car's heading could then be compared to the calculated heading from GPS measurements.

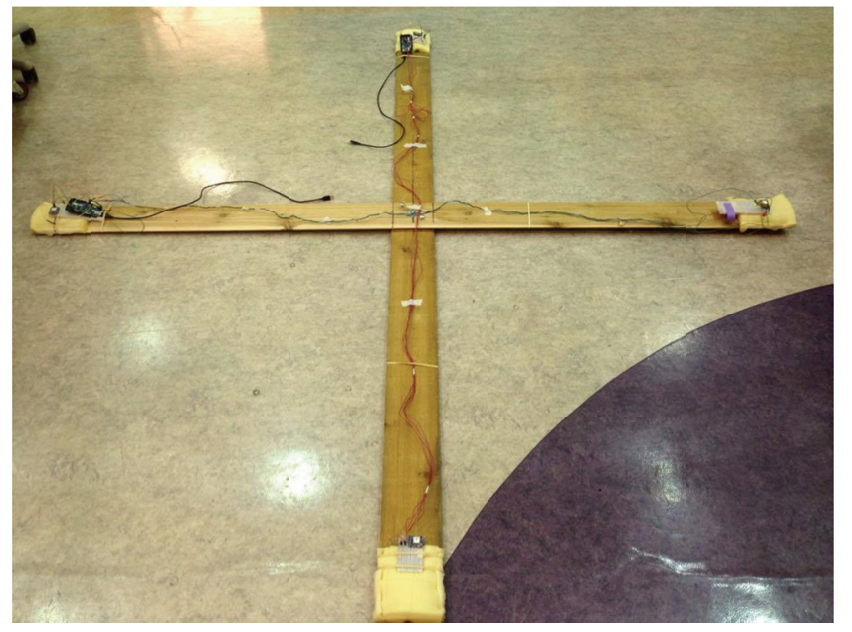

Fig. 2. The test hardware for the multiple-GPS method. At the end of each board is a GPS receiver connected to an Arduino Uno microcontroller. Positions are read out through a USB hub (not shown) to a computer for processing.

\section{B. Time-Differential Method}

If the vessel containing the antenna is moving in the direction its bow is facing, heading can be determined by looking at repeated measurements from a single GPS receiver. This method used an Arduino Uno microcontroller attached to an Adafruit Ultimate GPS Logger Shield. Once per second, the current position was stored and the angle between the current position and the previous position was calculated as the heading. If the GPS unit lacked a satellite fix or recorded the same position twice, the previous heading was returned.

This method does not give accurate results if the vessel is standing still, rotating in place, or drifting in a direction the bow is not facing. We designed algorithms to account for some of these situations by using a small gyroscope, but were not able to fully test the solutions. An automatic gain-based correction (see Discussion) might also account for these situations well.

\section{Motorized Antenna Mount}

Once the heading was calculated by either method, the Python module PyEphem calculated the current position of the satellite from its two-line element and the necessary altitude and azimuth for the antenna. The microcontroller sent instructions to two stepper motors that adjusted the azimuth and elevation of the antenna. The position of the antenna was updated every five seconds.

\section{Gain-Based Correction}

The Arduino microcontroller also received the signal gain from the satellite modem's automatic gain control (AGC) output, allowing it to measure the strength of the signal. If the gain dropped too low, readouts from the modem's AGC could be used to maintain lock on the satellite by adjusting the azimuth and altitude to maximize gain. The antenna could then be pointed in the direction where the gain is the highest. This method compensates for failures in the heading algorithms. However, technical difficulties prevented us from fully testing this system. 


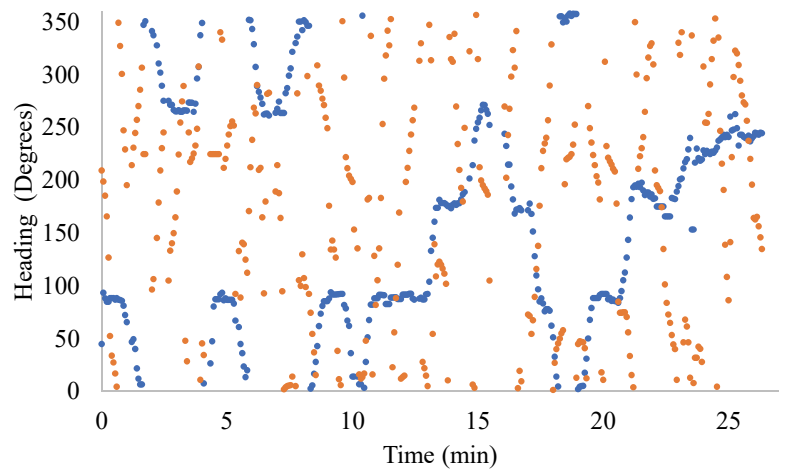

Fig. 3. Heading measured by two different methods on a test course. The multi-GPS method uses differences between four GPS receivers mounted on the vertices of a square of side $2.5 \mathrm{~m}$. The time-differential method examines consecutive measurements by a single GPS receiver. Equipment for both methods were placed on a car and driven around a calibrated test course. The time-differential method gave results that closely match the course, while the multi-GPS method's results appear to be randomly scattered.

\section{TESTING}

\section{A. Multiple-GPS Method}

We tested both methods by driving the equipment around a calibrated test course in a car. The heading values given by the time-differential method lined up well with the expected course directions; however, the multiple-GPS method gave heading values which varied erratically (Fig. 3). As a result, we deemed that this method is not viable for the antennas and distances we were using.

The underlying cause for the inaccurate heading values seems to be non-correlated systematic errors in the GPS units themselves. When two GPS units were placed directly next to one another, the average recorded distance was stable but was measured to be $1.42 \mathrm{~m}$. Repeated tests found that two units did not give a reliably different position until they were $18 \mathrm{~m}$ apart. This implies that each module varied substantially in their independently reported position. These additional errors rendered the heading algorithm unable to reliably fit the four points of the polygon using chi-squared values, which led to rapid fluctuations of the heading output. Although atmospheric effects also affected the measured positions, the changes were correlated between the modules, so the effect on heading determination was small.

\section{B. Time-Differential Method}

As Fig. 3 indicates, the time-differential method performed well at determining bearing on a calibrated test course. To test its performance with actual satellite communications, we used the US Electrodynamics, Inc. (USEI) teleport in Brewster, WA to transmit and receive the signal using the Omnispace F2 satellite. The vessel was simulated using a car with the mobile antenna mount attached to the top. We measured the $E_{b} / N_{0}$ from the satellite transmission as we drove along a simple test route.

For verification, we first drove the route using a commercial differential GPS unit by Hemisphere Global Navigation \& Surveillance Systems, which reliably determines heading to within a few degrees. Fig. 4(a) shows that $E_{b} / N_{0}$

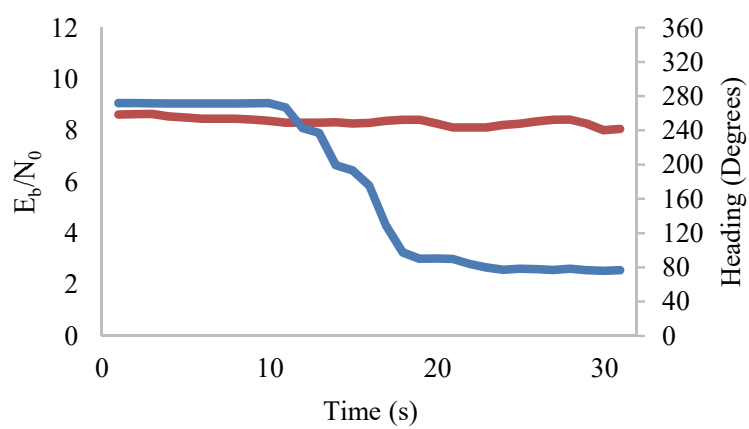

응

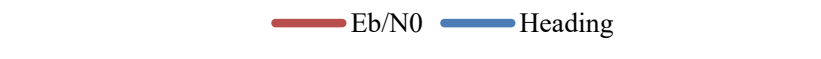

(a)

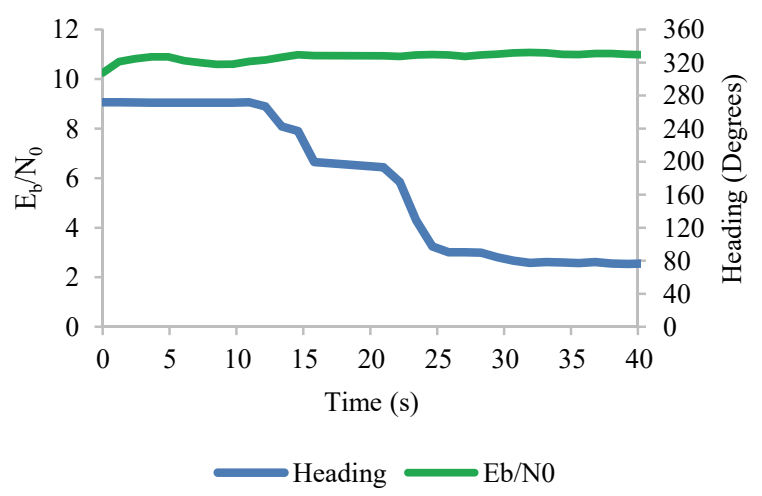

(b)

Fig. 4. $\mathrm{E}_{\mathrm{b}} / \mathrm{N}_{0}$ and heading data when using different methods to orient the satellite antenna. A motorized satellite antenna mount was mounted to the top of a car and driven on a simple test course. The direction of the satellite antenna was determined by (a) a high-end commercial orientation unit and (b) a low-cost time-differential method. Both algorithms maintained high $\mathrm{E}_{\mathrm{b}} / \mathrm{N}_{0}$ throughout the test. The differences in $\mathrm{E}_{\mathrm{b}} / \mathrm{N}_{0}$ between the two tests is because they were performed at different times during the satellite pass.

stays high and stable along the test route. We then repeated the test using the time-differential method, with results shown in Fig. 4(b). While the systems did not always agree on the heading (Fig. 5), the calculated heading was close enough to maintain a good $\mathrm{E}_{\mathrm{b}} / \mathrm{N}_{0}$. (The difference in $\mathrm{E}_{\mathrm{b}} / \mathrm{N}_{0}$ between the two is because they were conducted at different times during the satellite pass.)

\section{CONCLUSION}

Non-correlated systematic differences between GPS antennas prevent a multiple-GPS solution from being practical for low-cost receivers. More sophisticated solutions or higherquality antennas are required for a multiple-receiver solution. However, this study indicates that using low-cost GPS modules with a time-differential method is feasible for determining heading.

While the differential solution was able to maintain a high $\mathrm{E}_{\mathrm{b}} / \mathrm{N}_{0}$ for much of the test course, notable divergences from the actual heading occurred (Fig. 5). One reason for this is that differential algorithm relies on sufficient distance between the 


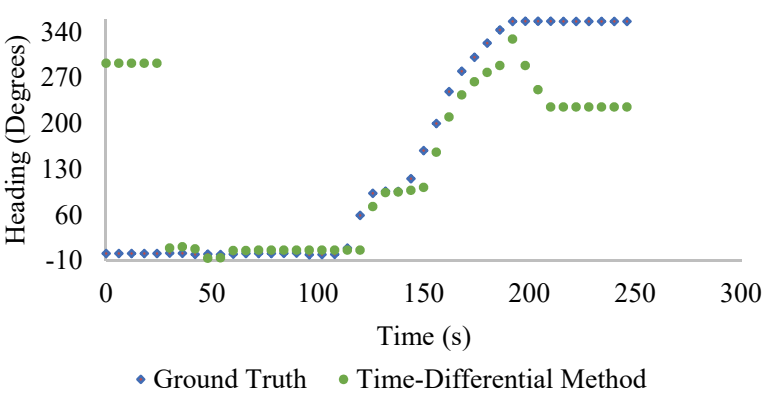

Fig. 5. Comparison in heading between the low-cost time-differential method and ground truth as measured by a commercial orientation unit. Both units were mounted on a car that drove around a simple test course. The two line up well for most of the course but diverge at the end.

two points to obtain an accurate heading. Because the polling rate for the GPS unit was $1 \mathrm{~Hz}$, the accuracy of the differential algorithm declined when the speed of the car was below $1 \mathrm{~m} / \mathrm{s}$. This can be solved by reducing the polling rate.

In conclusion, we showed that low-cost hardware may be realistically used to orient a satellite antenna to maintain connection with a medium earth orbit satellite from a moving vessel. While several issues with hardware and software remain to be resolved, the tests show a proof of principle that a high-quality signal can be maintained using a time-differential system. The gain-based correction system that was not tested should provide an additional layer of security to keep signal strength high. Further work will continue in this promising direction.

\section{ACKNOWLEDGMENTS}

We would like to thank Hector Correa for writing much of the code for the microcontrollers. Ryan Johnson and John Lin contributed substantially in the early stages of this investigation. USEI allowed us to uplink from their teleport and supported us with their facilities, supplies, expertise, and lots of food for the duration of the testing period. Tom Cox of USEI worked closely with us to ensure a successful test. This project was made possible by the Student Inquiry and Research program at the Illinois Mathematics and Science Academy under the direction of Sanza Kazadi.

\section{REFERENCES}

[1] Hemisphere. (2016, September). Vector V102 GPS Compass [Online]. Available:https://hemispheregnss.com/DesktopModules/EasyDNNNews /DocumentDownload.ashx?portalid=0\&moduleid=519\&articleid=19\&d ocumentid $=590$

[2] P. J. G. Teunissen and A. Khodabandeh, "Review and principles of PPPRTK methods," J Geod, vol. 89, no. 3, pp. 217-240, Nov. 2014. 